\title{
Clinical and Radiological Outcomes after Microscopic Bilateral Decompression via a Unilateral Approach for Degenerative Lumbar Disease: Minimum 5-Year Follow-Up
}

\author{
Sho Dohzono ${ }^{1}$, Hiromitsu Toyoda ${ }^{1}$, Akira Matsumura ${ }^{2}$, \\ Hidetomi Terai ${ }^{1}$, Akinobu Suzuki ${ }^{1}$, Hiroaki Nakamura ${ }^{1}$ \\ ${ }^{1}$ Department of Orthopaedic Surgery, Osaka City University Graduate School of Medicine, Osaka, Japan \\ ${ }^{2}$ Department of Orthopaedic Surgery, Osaka City General Hospital, Osaka, Japan
}

\begin{abstract}
Study Design: A retrospective study.
Purpose: To assess postoperative bone regrowth at surgical sites after lumbar decompression with $>5$ years of follow-up. Postoperative preservation of facet joints and segmental spinal instability following surgery were also evaluated.

Overview of Literature: Previous reports have documented bone regrowth after conventional laminectomy or laminotomy and several factors associated with new bone formation.

Methods: Forty-nine patients who underwent microscopic bilateral decompression via a unilateral approach at L4-5 were reviewed. Primary outcomes included correlations among postoperative bone regrowth, preservation of facet joints, radiographic parameters, and clinical outcomes. Secondary outcomes included comparative analyses of radiographic parameters and clinical outcomes among preoperative diagnoses (lumbar spinal stenosis, degenerative spondylolisthesis, and degenerative lumbar scoliosis).

Results: The average value of bone regrowth at the latest follow-up was significantly higher on the dorsal side of the facet joint (3.4 $\mathrm{mm})$ than on the ventral side $(1.3 \mathrm{~mm})$. Percent facet joint preservation was significantly smaller on the approach side $(79.2 \%)$ than on the contralateral side (95.2\%). Bone regrowth showed a significant inverse correlation with age, but no significant correlation was observed with facet joint preservation, gender, postoperative segmental spinal motion, or clinical outcomes. Subanalysis of these data revealed that bone regrowth at the latest follow-up was significantly greater in patients with degenerative lumbar scoliosis than in those with lumbar spinal stenosis. Postoperative segmental spinal motion at $L 4-L 5$ did not progress significantly in patients with degenerative spondylolisthesis or degenerative lumbar scoliosis compared with those with lumbar spinal stenosis.

Conclusions: Microscopic bilateral decompression via a unilateral approach prevents postoperative spinal instability because of satisfactory preservation of facet joints, which may be the primary reason for inadequate bone regrowth. Postoperative bone regrowth was not related to clinical outcomes and postoperative segmental spinal instability.
\end{abstract}

Keywords: Lumbar osteoarthritis; Treatment efficacy; Bone development; Minimally invasive surgery

\footnotetext{
Received Jun 15, 2016; Revised Jul 22, 2016; Accepted Jul 28, 2016

Corresponding author: Hiromitsu Toyoda

Department of Orthopaedic Surgery, Osaka City University Graduate School of Medicine,

1-4-3 Asahimachi, Abeno-ku, Osaka 545-8585, Japan

Tel: +?81-6-6645-3851; Fax: +81-6-6646-6260, E-mail: h-toyoda@msic.med.osaka-cu.ac.jp
} 


\section{Introduction}

Postoperative spinal instability, particularly segmental spinal instability, affects clinical outcomes after decompression surgery. To preserve the facet joint and paraspinal muscles, certain studies have reported minimally invasive decompression surgery with a microscope or microendoscope [1-3]. However, little is known about the relationship between postoperative spinal instability and facet joint preservation. On the other hand, moderate and marked postoperative bone regrowth may be related to recurrence of neurological symptoms in the mid and long terms [4]. Bone regrowth at the surgical site is considered to represent the natural history of bone healing. Previous studies have documented bone regrowth after conventional laminectomy or laminotomy and several factors associated with new bone formation [4-6]. However, the type of surgery, preoperative diagnosis, duration of followup, and method of measurement could affect the amount of bone regrowth and degree of postoperative vertebral stability. The purpose of our study was to assess midterm outcomes after microscopic bilateral decompression via a unilateral approach (MBDU) in terms of postoperative bone regrowth and preservation of facet joints in patients with degenerative lumbar disease.

\section{Materials and Methods}

This study was approved by the Institutional Review Board of Osaka City University Graduate School of Medicine.

Ninety patients who underwent MBDU at lumbar vertebra 4 (L4)-L 5 between 2001 and 2008 at our institute were retrospectively reviewed. All patients had presented with lower extremity pain and/or numbness. The radiographic indications for this surgical procedure were lumbar spinal stenosis (LSS) without instability, degenerative spondylolisthesis (DS) with percent slip $<25 \%$, segmental kyphosis in flexion $<5^{\circ}$, angular motion of the disc $<10^{\circ}$, and degenerative lumbar scoliosis (DLS). Exclusion criteria among patients with DLS were as follows: (1) Cobb angle $>25^{\circ}$, (2) severe low back pain, (3) changes in segmental disc wedging between the standing and prone positions $>$ $5^{\circ}$, (4) lateral disc slippage $>3 \mathrm{~mm}$, and (5) foraminal stenosis requiring $>50 \%$ facetectomy for decompression [7].

Of the 90 patients, nine patients with other neurological symptom (eight with cervical myelopathy and one with Parkinson's disease) were excluded from the analysis.
Table 1. Patients' characteristics

\begin{tabular}{lc} 
Characteristic & Value \\
\hline Average age (yr) & $67.9 \pm 8.4$ \\
\hline Sex (male/female) & $28 / 21$ \\
\hline Average follow-up (yr) & $6.7 \pm 1.9$ \\
\hline Diagnosis & 20 \\
\hline Lumbar spinal canal stenosis & 14 \\
\hline Degenerative lumbar spondylolisthesis & 15 \\
\hline Degenerative lumbar scoliosis & $22 / 20 / 6 / 1$ \\
\hline No. levels decompressed (1/2/3/4)
\end{tabular}

Of the 81 patients, 49 patients with follow-up data for $>5$ years were retrospectively reviewed (follow-up rate, $60.5 \%)$. There were 28 men and 21 women, aged $49-82$ years (average, 67.9 years) with a follow-up duration of 5-13 years (mean, 6.6 years). Preoperative diagnoses were LSS in 20 patients, DS in 14 patients, and DLS in 15 patients. Single-level, two-level, three-level, and fourlevel decompressions were performed in 22, 20,6, and 1 patient, respectively (Table 1).

\section{Outcome measures}

The primary outcome was correlation between radiographic parameters and clinical outcomes after MBDU. The secondary outcome was comparative analyses of radiographic parameters and clinical outcomes in terms of preoperative diagnoses (LSS, DS, and DLS).

\section{Surgical procedures}

All patients underwent MBDU to decompress the central canal and bilateral lateral recesses, performed as previously described $[7,8]$. A midline incision of approximately $35 \mathrm{~mm}$ was made for single decompression to unilaterally expose the posterior elements lateral to the facet joints. Microscopic laminotomy was performed on the approach side using an air drill, Kerrison rongeur, and microcurette. Subsequently, decompression was performed on the contralateral side after rotating the operating table to the contralateral side.

\section{Clinical evaluations}

Clinical outcomes were evaluated using the Japanese 
Orthopaedic Association (JOA) scores for low back pain preoperatively, 2 years postoperatively, and at the latest follow-up. The improvement rate for the JOA scores was calculated as (postoperative JOA score-preoperative JOA score $) /(29$-preoperative JOA score $) \times 100(\%)$.

\section{Radiographic evaluations}

On lateral radiographs, percent slip in the neutral position, dynamic percent slip, and angular motion of the disc between the flexion and extension positions at L4-L5 were measured preoperatively, at 2 years postoperatively, and at the latest follow-up. On anteroposterior plain radiographs, the scoliotic wedging angle at L4-L5 was measured preoperatively, at 2 years postoperatively, and at the latest follow-up. Percent facet joint preservation was calculated on the approach and contralateral sides using the following equation: (postoperative width of the facet/ preoperative width of the facet) $\times 100$ (Fig. 1).
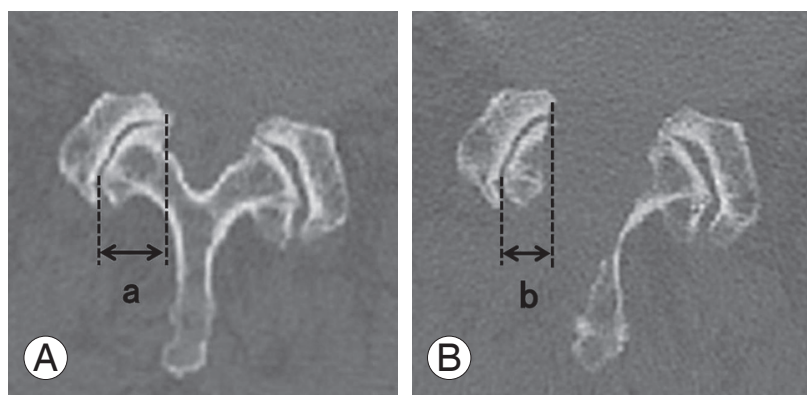

Fig. 1. Percentage of facet joint preservation, measured on axial computed tomography images at $L 4-L 5$ preoperatively $(\mathbf{A})$ and immediately postoperatively $(B)$. Percentage of facet joint preservation= $\mathrm{b} / \mathrm{a} \times 100$.

\section{Bone regrowth}

Bone regrowth was assessed on computed tomography (CT) (Somatom Sensation 64 Cardiac, Siemens, Germany) at 1 week postoperatively, at 2 years postoperatively, and at the latest follow-up. Average timing of CT at the latest follow-up was 6 years. As previously reported [9], decrease in the postoperative defect of the lamina and facet joint was defined as bone regrowth. Bone regrowth was calculated on the ventral and dorsal sides of the facet joint at 2 years postoperatively and at the final follow-up. Fig. 2 illustrates the method used for measuring bone regrowth.

\section{Statistical analysis}

IBM SPSS ver. 19 (IBM Corp., Armonk, NY, USA) was used for all statistical analyses, and a $p$-value of $<0.05$ was considered significant. To evaluate the reliability of techniques used to measure facet joint preservation and bone regrowth, we evaluated the intraobserver and interobserver variabilities. Two authors (S.D. and H.T.), each with $>10$ years of experience and blinded to patients' clinical variables, independently measured the parameters. After each author had measured a parameter on a radiograph twice, the intraobserver and interobserver variabilities of the average values were evaluated as intraclass coefficient of correlation (ICC). Correlations were analyzed using Pearson's correlation test. Multiple comparison analyses of radiographic parameters among preoperative diagnoses were performed using the Tukey-Kramer test for parametric data or the Kruskal-Wallis test for nonparametric data.
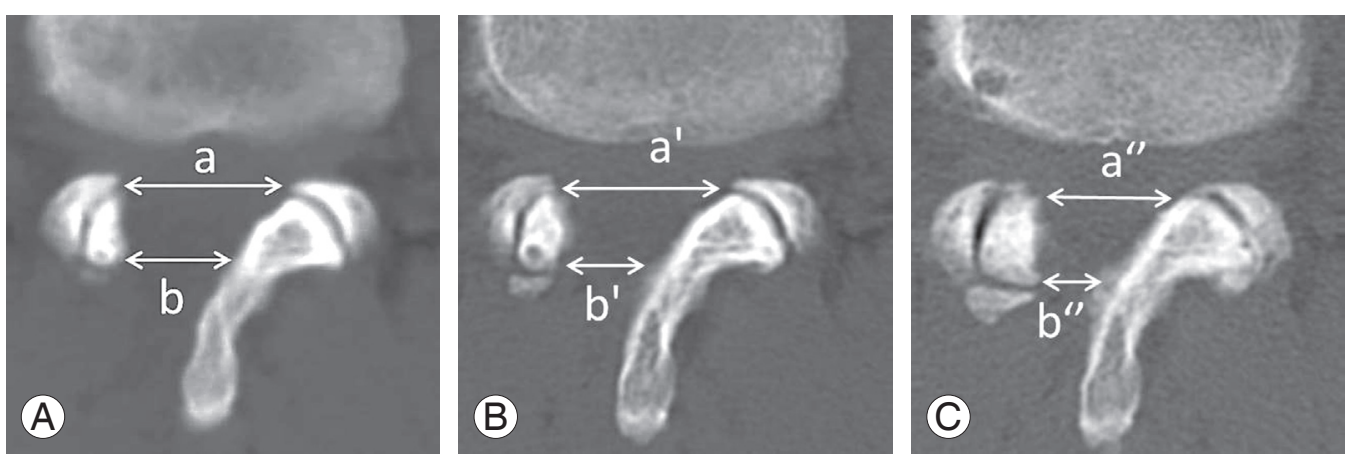

Fig. 2. Axial computed tomography at $L 4-L 5$ recorded 1 week postoperatively (A), 2 years postoperatively (B), and at the latest follow-up (C). Bone regrowth $(\mathrm{mm})=($ distance 1 week postoperatively)-(postoperative distance). a, a', a", ventral side of the facet; $b, b^{\prime}, b^{\prime \prime}$, dorsal side of the facet. 


\section{Results}

Reliability of the radiological measurements of facet joint preservation and bone regrowth were confirmed by calculating intraobserver and interobserver variabilities of each parameter. The intraobserver ICC values of all parameters were $>0.92$ and the interobserver ICC values were 0.87 , indicating excellent reproducibility of measurements [10].

\section{Clinical outcomes}

The average JOA score was $12.9 \pm 3.5$ points preoperatively, which improved to $24.9 \pm 3.2$ points at 2 years postoperatively and to $23.3 \pm 4.8$ points at the latest followup. The average improvement rate of JOA scores was $66.2 \% \pm 27.6 \%$ at 2 years postoperatively and $65.8 \% \pm 25.7 \%$

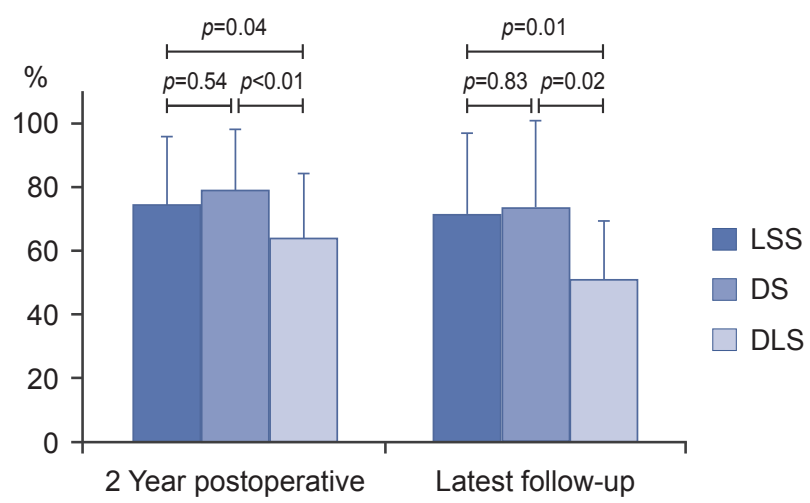

Fig. 3. Average improvement rate of JOA scores at 2 years postoperatively and the latest follow-up in patients with LSS, DS, and DLS. LSS, lumbar spinal stenosis; DS, degenerative spondylolisthesis; DLS, degenerative lumbar scoliosis; JOA, Japanese Orthopaedic Association. at the latest follow-up. On evaluation by preoperative diagnosis, patients with LSS and DS had average improvement rates of $74.6 \%$ and $79.1 \%$, respectively, at 2 years postoperatively, and of $71.6 \%$ and $73.5 \%$, respectively, at the latest follow-up. There were no significant differences in JOA scores between LSS and DS; however, these scores for patients with DLS were $54.1 \%$ and $50.9 \%$, respectively, which were significantly lower than those for LSS and DS (Fig. 3).

\section{Primary outcomes}

Percent facet joint preservation was significantly lower on the approach side than on the contralateral side $(79.2 \% \pm 16.2 \%$ vs. $95.2 \% \pm 7.6 \%$; $p<0.01)$. Table 2 summarizes the primary outcomes in the correlation coefficients between facet joint preservation and radiological parameters. Facet joint preservation was not significantly correlated with the difference between preoperative and postoperativelpercent slip in the neutral position, dynamic percent slip, angular motion of the disc, scoliotic wedging angle at L4-L5, or the improvement rate of JOA scores.

The average amount of bone regrowth was $2.5 \pm 1.6 \mathrm{~mm}$ at 2 years postoperatively and $3.4 \pm 2.1 \mathrm{~mm}$ at the latest follow-up on the dorsal side of the facet joint and $1.0 \pm 1.2$ $\mathrm{mm}$ at 2 years postoperatively and $1.3 \pm 1.3 \mathrm{~mm}$ at the latest follow-up on the ventral side. The average bone regrowth at the latest follow-up was significantly greater on the dorsal side than on the ventral side of the facet joint. Table 3 presents primary outcomes of correlation coefficients between bone regrowth and radiological pa-

Table 2. Correlation coefficients between facet joint preservation and radiologic parameters

\begin{tabular}{|c|c|c|c|c|c|}
\hline \multirow{2}{*}{ Parameter } & \multirow{2}{*}{ Mean \pm SD } & \multicolumn{2}{|c|}{$\begin{array}{l}\text { Facet joint preservation } \\
\text { (approach side) }\end{array}$} & \multicolumn{2}{|c|}{$\begin{array}{l}\text { Facet joint preservation } \\
\text { (contralateral side) }\end{array}$} \\
\hline & & $\begin{array}{l}\text { Correlation } \\
\text { coefficient }\end{array}$ & $p$-value & $\begin{array}{l}\text { Correlation } \\
\text { coefficient }\end{array}$ & $p$-value \\
\hline Age (yr) & $67.9 \pm 8.4$ & 0.049 & 0.743 & -0.092 & 0.538 \\
\hline$\Delta \%$ slip in neutral position (\%) & $2.6 \pm 2.7$ & -0.108 & 0.570 & 0.111 & 0.558 \\
\hline $\begin{array}{l}\Delta \text { dynamic } \% \text { slip between flexion and } \\
\text { extension }(\%)\end{array}$ & $2.1 \pm 2.2$ & 0.143 & 0.451 & 0.006 & 0.973 \\
\hline$\Delta$ angular motion of the disc $\left(^{\circ}\right)$ & $-1.0 \pm 5.1$ & -0.027 & 0.885 & -0.112 & 0.557 \\
\hline$\Delta$ scoliotic wedging angle at $L 4 / 5\left(^{\circ}\right)$ & $3.9 \pm 4.3$ & 0.089 & 0.626 & -0.065 & 0.726 \\
\hline JOA improvement (\%) & $65.8 \pm 25.7$ & -0.086 & 0.567 & -0.100 & 0.505 \\
\hline
\end{tabular}

SD, standard deviation; $\triangle$, (value at latest follow-up)-(value before surgery); JOA, Japanese Orthopaedic Association. 
Table 3. Correlation coefficients between bone regrowth and radiologic parameters

\begin{tabular}{|c|c|c|c|c|c|}
\hline \multirow{2}{*}{ Parameter } & \multirow{2}{*}{ Mean \pm SD } & \multicolumn{2}{|c|}{$\begin{array}{l}\text { Bone regrowth } \\
\text { (ventral side) }\end{array}$} & \multicolumn{2}{|c|}{$\begin{array}{l}\text { Bone regrowth } \\
\text { (dorsal side) }\end{array}$} \\
\hline & & $\begin{array}{l}\text { Correlation } \\
\text { coefficient }\end{array}$ & $p$-value & $\begin{array}{l}\text { Correlation } \\
\text { coefficient }\end{array}$ & $p$-value \\
\hline Age $(y r)$ & $67.9 \pm 8.4$ & -0.262 & 0.069 & -0.256 & 0.076 \\
\hline No. levels decompressed & $1.7 \pm 0.8$ & 0.142 & 0.331 & -0.035 & 0.809 \\
\hline Facet joint preservation (approach side) (\%) & $79.2 \pm 16.2$ & -0.027 & 0.859 & -0.081 & 0.589 \\
\hline Facet joint preservation (contralateral side) (\%) & $95.2 \pm 7.6$ & -0.213 & 0.150 & -0.024 & 0.875 \\
\hline$\Delta \%$ slip in neutral position (\%) & $2.6 \pm 2.7$ & 0.200 & 0.289 & 0.148 & 0.437 \\
\hline $\begin{array}{l}\Delta \text { dynamic } \% \text { slip between flexion and exten- } \\
\operatorname{sion}(\%)\end{array}$ & $2.1 \pm 2.2$ & -0.015 & 0.936 & -0.045 & 0.813 \\
\hline$\Delta$ angular motion of the disc $(\%)$ & $-1.0 \pm 5.1$ & 0.024 & 0.895 & 0.044 & 0.803 \\
\hline$\Delta$ scoliotic wedging angle at $L 4 / 5(\%)$ & $3.9 \pm 4.3$ & -0.003 & 0.990 & 0.286 & 0.125 \\
\hline JOA improvement (\%) & $65.8 \pm 25.7$ & 0.102 & 0.485 & -0.017 & 0.910 \\
\hline
\end{tabular}

$\mathrm{SD}$, standard deviation; $\Delta$, (value at latest follow-up)-(value before surgery).

Table 4. Changes in radiographic parameters during follow-up in patients with LSS, DS, and DLS

\begin{tabular}{|c|c|c|c|c|}
\hline Parameter & LSS & DS & DLS & $p$-value \\
\hline Age & $64.8 \pm 8.6$ & $70.0 \pm 8.5$ & $70.1 \pm 7.0$ & 0.095 \\
\hline \multicolumn{5}{|c|}{ Percent slip in the neutral position } \\
\hline Before surgery & 0.35 & 9.91 & 1.42 & $<0.01^{\mathrm{a})}$ \\
\hline 2 Years postoperative & 1.04 & 10.59 & 1.08 & $<0.01^{\text {a) }}$ \\
\hline Latest follow-up & 1.16 & 11.59 & 1.07 & $<0.01^{\text {a) }}$ \\
\hline \multicolumn{5}{|c|}{ Dynamic percent slip between flexion and extension } \\
\hline Before surgery & 2.40 & 2.73 & 1.24 & 0.35 \\
\hline 2 Years postoperative & 2.63 & 3.38 & 1.58 & 0.39 \\
\hline Latest follow-up & 2.77 & 4.30 & 3.47 & 0.49 \\
\hline \multicolumn{5}{|l|}{ Angular motion of the disc } \\
\hline Before surgery & 6.60 & 6.61 & 7.74 & 0.68 \\
\hline 2 Years postoperative & 6.00 & 5.73 & 4.18 & 0.51 \\
\hline Latest follow-up & 5.78 & 4.77 & 2.92 & 0.23 \\
\hline \multicolumn{5}{|c|}{ Scoliotic wedging angle at $L 4 / 5$} \\
\hline Before surgery & 0.82 & 1.25 & 3.81 & $<0.01^{\text {a) }}$ \\
\hline 2 Years postoperative & 1.55 & 0.92 & 4.71 & $<0.01^{\text {a) }}$ \\
\hline Latest follow-up & 1.74 & 1.84 & 5.97 & $<0.01^{\text {a) }}$ \\
\hline
\end{tabular}

Values are means \pm standard deviation.

LSS, lumbar spinal stenosis; DS, degenerative spondylolisthesis; DLS, degenerative lumbar scoliosis.

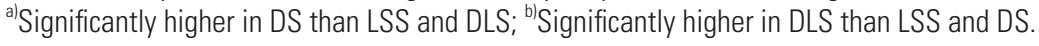

rameters. Bone regrowth was inversely correlated with age (dorsal side/ventral side: $r=-0.256 /-0.261 ; p=0.076 / 0.070$, respectively). Moreover, bone regrowth was not significantly correlated with percent facet joint preservation and change in preoperative and postoperative percent slip in the neutral position, dynamic percent slip, angular motion of the disc, scoliotic wedging angle at L4-L5, and the improvement rate of JOA scores. 


\section{Secondary outcomes (comparative analyses among preoperative diagnoses)}

Table 4 shows changes in radiographic parameters during follow-up in patients with LSS, DS, and DLS. The preoperative percentages of anterior slip in patients with LSS, DS, and DLS were $0.4 \% \pm 0.9 \%, 9.9 \% \pm 3.9 \%$, and $1.4 \% \pm 4.3 \%$, respectively, and the percentages of anterior slip at the latest follow-up were $1.2 \% \pm 4.8 \%, 11.6 \% \pm 4.4 \%$, and $1.1 \% \pm 2.5 \%$, respectively. No significant differences were observed in the progression of anterior slip among these diseases. The preoperative dynamic percentage slips between flexion and extension positions in patients with LSS, DS, and DLS were $2.4 \% \pm 2.9 \%, 2.7 \% \pm 1.9 \%$, and $1.2 \% \pm 2.7 \%$, respectively, and those at the latest follow-up were $2.8 \% \pm 2.3 \%, 4.3 \% \pm 3.8 \%$, and $3.5 \% \pm 4.1 \%$, respectively. Thus, there were no significant differences in dynamic percentage slip among these diseases. In addition, there were no significant differences in angular motion of the disc among these diseases. The preoperative scoliotic wedging angles at L4-L5 in patients with LSS, DS, and DLS were $0.8^{\circ} \pm 0.5^{\circ}, 1.3^{\circ} \pm 1.1^{\circ}$, and $3.8^{\circ} \pm 2.7^{\circ}$, respectively, whereas these angles at the latest follow-up were $2.2^{\circ} \pm 2.2^{\circ}$, $1.8^{\circ} \pm 1.6^{\circ}$, and $6.0^{\circ} \pm 3.7^{\circ}$, respectively. Thus, there were no significant differences in the progression of scoliotic wedging angle at L4-L5 among these diseases.

Percent facet joint preservation on the approach side was significantly smaller in patients with DS (70.0\%) than in those with LSS and DLS (83.2\% and $82.5 \%$, respectively). On the other hand, facet joint preservation on the contralateral side was not significantly different among

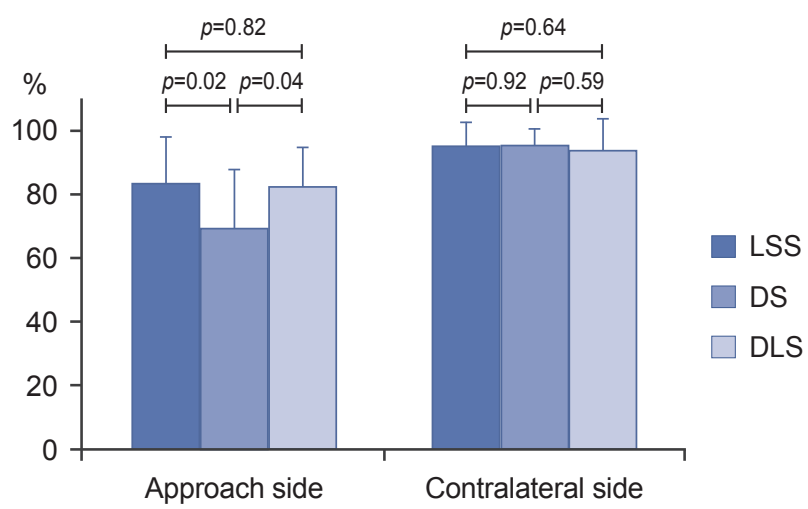

Fig. 4. Percentage of facet joint preservation on the approach and contralateral sides in patients with LSS, DS, and DLS. LSS, lumbar spinal stenosis; DS, degenerative spondylolisthesis; DLS, degenerative lumbar scoliosis. patients with LSS, DS, and DLS (94.7\%, 95.8\%, and 95\%, respectively) (Fig. 4). Bone regrowth at the latest followup was significantly higher in patients with DLS than in those with LSS and DS on the ventral sides of the facet joint and higher than those with LSS on the dorsal side of the facet joint (Fig. 5).

\section{Discussion}

We previously reported that the amount of bone regrowth after MBDU was relatively smaller in patients with DS and LSS at a follow-up of at least 2 years [9]. However, little is known about the relationship between facet joint preservation and postoperative spinal instability, long-term bone regrowth, and factors associated with bone regrowth.

Here MBDU preserved $70 \%-83 \%$ of the facet joint on the approach side and $>90 \%$ of the facet joint on the contralateral side. MBDU reduced the risk of postoperative spinal instability (percent slip and scoliotic wedging angle) at the surgical site, indicated by satisfactory preservation of the facet joint. Postoperative bone regrowth was not related to clinical outcomes and postoperative segmental spinal instability at a follow-up of at least 5 years.

\section{Clinical outcomes in patients with DLS}

The improvement rate for DLS in the present study was $54.1 \%$ at 2 years postoperatively and $50.9 \%$ at the latest follow-up, and these were significantly lower than improvement rates for LSS and DS. This recovery rate for DLS was similar to that reported in previous studies $[7,8]$

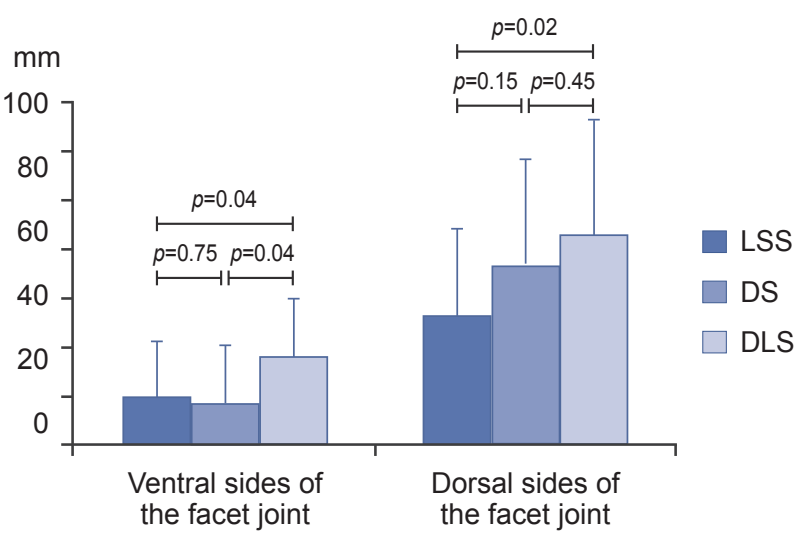

Fig. 5. Average value of bone regrowth at the latest follow-up on the dorsal and ventral sides of the facet joint in patients with LSS, DS, and DLS. LSS, lumbar spinal stenosis; DS, degenerative spondylolisthesis; DLS, degenerative lumbar scoliosis. 
and is thus considered acceptable. However, re-surgery rates for patients with DLS were relatively higher than those for patients without scoliosis, even with minimally invasive procedures [11]. Therefore, it should be carefully determined whether this procedure should be used for treatment of DLS.

\section{Facet joint preservation}

During lumbar decompression surgery, the extent of preservation of the posterior element is an important factor for achieving good surgical results. A morphological study revealed that patients with DS exhibited greater sagittal orientation of the facet joint [12]. Thus, after decompression, patients with DS would have decreased ability to resist anterior shear forces than would those without DS. To preserve facet joint and paraspinal muscles, certain procedures, such as lumbar spinous process-splitting laminectomy [13], muscle-preserving interlaminar decompression with a microscope or microendoscope $[1,2]$, and microendoscopic decompression via the paramedian approach, have been suggested [3]

Here the average percent facet joint preservation on the approach side was significantly smaller in DS (70.0\%) than in LSS and DLS (83.2\% and $82.5 \%$, respectively). Miyazaki et al. [14] reported that patients with DS were associated with sagittally oriented facets and narrow osseous canals. Therefore, we speculate that it is technically more difficult to preserve facet joint on the approach side in patients with DS than in those without DS. However, here the average facet joint preservation on the contralateral side was $>90 \%$ regardless of preoperative diagnosis. Thus, segmental spinal instability at the latest follow-up, such as percent slip in the neutral position, dynamic percent slip, and angular motion of the disc, did not progress significantly in patients with DS compared with those with LSS.

\section{Radiological changes at L4-L5 postoperatively}

One of the reasons a surgeon may hesitate to perform decompression alone in patients with DS or DLS is that there is limited information regarding progression of spinal instability after decompression surgery. Here percent slip in the neutral position postoperatively for patients with DS increased from $9.9 \%$ preoperatively to $10.6 \%$ at 2 years postoperatively and $11.6 \%$ at the latest follow-up. The percent slip progressed in a time-dependent man- ner; however, it was as slight as previously reported, with a follow-up of at least 2 years [15]. Similarly, in patients with DLS, the scoliotic wedging angle at L4-L5 postoperatively increased in a time-dependent manner, from $3.8^{\circ}$ preoperatively to $4.7^{\circ}$ at 2 years postoperatively and $6.0^{\circ}$ at the latest follow-up. Hosogane et al. [16] reported that the curve progression after decompression alone in patients with mild DLS was $1.4^{\circ}$ per year, which was almost equivalent to that occurring during the natural course of DLS. Here average progression of the scoliotic wedging angle at L4-L5 was $0.4^{\circ}$ per year. Thus, it appears that local curve progression after decompression for DLS is as slight as that during the natural course of DLS.

\section{Bone regrowth}

Bone regrowth at the surgical site is considered to represent the natural history of bone healing. For evaluation of bone regrowth, Postacchini and Cinotti [6] used only plain radiographs, wherein 16 of 74 decompression levels revealed moderate or marked bone regrowth. Moreover, Chen et al. [4] used plain radiographs alone and reported moderate or marked bone regrowth in $>40 \%$ of patients. Narrowing of the spinal canal by bone regrowth is primarily caused at the facet joint level, and regrowth of the lamina arc does not usually cause marked compression, except in DS [6]. Guigui et al. [5] recommended the use of $\mathrm{CT}$ to estimate bone regrowth because plain radiographs overestimate it in comparison with CT. Hence, here, bone regrowth was calculated using $\mathrm{CT}$ on the ventral and dorsal sides of the facet joint to evaluate the lesion at the intracanal space and laminar arch.

Several factors associated with new bone formation have been reported. Chen reported that younger age $(<60$ years) was associated with considerably greater bone regrowth than older age ( $>60$ years). Similarly, here, bone regrowth was inversely correlated with age. However, certain studies have reported lack of correlation between bone regrowth and age. Thus, the relationship between age and bone regrowth remains controversial.

Several studies have reported that postoperative destabilization increases bone regrowth [4-6]. Although we agree with this, we did not find a correlation between postoperative increase in percent slip and bone regrowth. This is because during surgery, there was only one patient whose vertebral slip progressed $>3 \mathrm{~mm}$, which was defined as postoperative destabilization in the previous study. Post- 
acchini and Cinotti [6] and Chen et al. [4] found a relationship between clinical results and the degree of bone regrowth. They found that poor outcomes were associated with a moderate or high degree of bone regrowth; however, contradictory to these results, we did not find an association of bone regrowth with restenosis and clinical outcomes. In comparison with previous studies in which conventional laminectomy or laminotomy was performed, bone regrowth associated with this procedure was lesser in our study.

Bone regrowth at the latest follow-up was significantly higher in patients with DLS than in those with LSS and DS. The pathophysiology of DLS is asymmetric degeneration of the disc and facet joint that leads to asymmetric loading of the lumbar spinal column, manifesting as a three-dimensional deformity. Such three-dimensional loading of the facet joint might have an effect on bone regrowth. The biological reaction to three-dimensional loading might have some effect on vertebral osteophyte formation as well as bone regrowth. However, this hypothesis remains unproven based on results of the present study. Therefore, additional studies focusing on the asymmetry of bone regrowth in patients with DLS should be performed to confirm this hypothesis.

Our study has several limitations. First, this was a retrospective study, and the indications for this surgical procedure were limited in patients with DS and DLS. Second, bone regrowth was measured at only one slice in the transverse direction. Thus, there is a potential for measurement errors in radiographic evaluations. Further research on three-dimensional bone regrowth formation might be warranted to obtain new information to add to the existing knowledge pool. Finally, the sample size in each group was not sufficient to yield substantial effects. Power analysis was performed using a software ( $G^{*}$ power 3.1.9.2: Faul, Erdfelder, Lung, \& Buchner, 2007) $[17,18]$. A total sample size of 64 patients was needed for correlation tests to obtain sufficient power $(0.8$, medium effect size and an $\alpha$-error probability of 0.05 ). Additional studies with larger samples and longer follow-up periods should be performed to confirm the present results.

\section{Conclusions}

MBDU prevents postoperative spinal instability owing to satisfactory preservation of the facet joint, which is considered the primary reason for the small amount of bone regrowth. Postoperative bone regrowth in this procedure was not related to clinical outcomes and postoperative segmental spinal instability.

\section{Conflict of Interest}

No potential conflict of interest relevant to this article was reported.

\section{References}

1. Hatta Y, Shiraishi T, Sakamoto A, et al. Muscle-preserving interlaminar decompression for the lumbar spine: a minimally invasive new procedure for lumbar spinal canal stenosis. Spine (Phila Pa 1976) 2009; 34:E276-80.

2. Mikami Y, Nagae M, Ikeda T, Tonomura H, Fujiwara $\mathrm{H}$, Kubo T. Tubular surgery with the assistance of endoscopic surgery via midline approach for lumbar spinal canal stenosis: a technical note. Eur Spine J 2013;22:2105-12.

3. Nomura K, Yoshida M. Microendoscopic decompression surgery for lumbar spinal canal stenosis via the paramedian approach: preliminary results. Global Spine J 2012;2:87-94.

4. Chen Q, Baba H, Kamitani K, Furusawa N, Imura S. Postoperative bone re-growth in lumbar spinal stenosis: a multivariate analysis of 48 patients. Spine (Phila Pa 1976) 1994;19:2144-9.

5. Guigui P, Barre E, Benoist M, Deburge A. Radiologic and computed tomography image evaluation of bone regrowth after wide surgical decompression for lumbar stenosis. Spine (Phila Pa 1976) 1999;24:281-8.

6. Postacchini F, Cinotti G. Bone regrowth after surgical decompression for lumbar spinal stenosis. J Bone Joint Surg Br 1992;74:862-9.

7. Matsumura A, Namikawa T, Terai H, et al. The influence of approach side on facet preservation in microscopic bilateral decompression via a unilateral approach for degenerative lumbar scoliosis: clinical article. J Neurosurg Spine 2010;13:758-65.

8. Toyoda H, Nakamura H, Konishi S, Dohzono S, Kato M, Matsuda H. Clinical outcome of microsurgical bilateral decompression via unilateral approach for lumbar canal stenosis: minimum five-year follow-up. Spine (Phila Pa 1976) 2011;36:410-5.

9. Dohzono S, Matsumura A, Terai H, Toyoda H, Su- 
zuki A, Nakamura H. Radiographic evaluation of postoperative bone regrowth after microscopic bilateral decompression via a unilateral approach for degenerative lumbar spondylolisthesis. J Neurosurg Spine 2013;18:472-8.

10. Landis JR, Koch GG. The measurement of observer agreement for categorical data. Biometrics 1977;33: 159-74.

11. Kelleher MO, Timlin M, Persaud O, Rampersaud YR. Success and failure of minimally invasive decompression for focal lumbar spinal stenosis in patients with and without deformity. Spine (Phila Pa 1976) 2010; 35:E981-7.

12. Grobler LJ, Robertson PA, Novotny JE, Ahern JW. Decompression for degenerative spondylolisthesis and spinal stenosis at L4-5. The effects on facet joint morphology. Spine (Phila Pa 1976) 1993;18:1475-82.

13. Watanabe K, Hosoya T, Shiraishi T, Matsumoto M, Chiba K, Toyama Y. Lumbar spinous process-splitting laminectomy for lumbar canal stenosis: technical note. J Neurosurg Spine 2005;3:405-8.

14. Miyazaki M, Morishita Y, Takita C, Yoshiiwa T, Wang
JC, Tsumura H. Analysis of the relationship between facet joint angle orientation and lumbar spine canal diameter with respect to the kinematics of the lumbar spinal unit. J Spinal Disord Tech 2010;23:242-8.

15. Sasai K, Umeda M, Maruyama T, Wakabayashi E, Iida H. Microsurgical bilateral decompression via a unilateral approach for lumbar spinal canal stenosis including degenerative spondylolisthesis. J Neurosurg Spine 2008;9:554-9.

16. Hosogane N, Watanabe K, Kono H, Saito M, Toyama Y, Matsumoto M. Curve progression after decompression surgery in patients with mild degenerative scoliosis. J Neurosurg Spine 2013;18:321-6.

17. Faul F, Erdfelder E, Buchner A, Lang AG. Statistical power analyses using $G^{*}$ Power 3.1: tests for correlation and regression analyses. Behav Res Methods 2009; 41:1149-60.

18. Faul F, Erdfelder E, Lang AG, Buchner A. G*Power 3: a flexible statistical power analysis program for the social, behavioral, and biomedical sciences. Behav Res Methods 2007;39:175-91. 